\title{
ON THE MAXIMUM MODULUS OF POLYNOMIALS
}

\author{
M. A. QAZI
}

(Communicated by Clifford J. Earle, Jr.)

\begin{abstract}
The maximum modulus on $|z|=r<1$ of a polynomial of degree $n$ not vanishing in $|z|<1$ is estimated in terms of its maximum modulus on $|z|=1$ and certain of its coefficients. Some other related problems are also considered.
\end{abstract}

\section{INTRODUCTION}

Let $\mathscr{P}_{n}$ denote the class of all polynomials of degree at most $n$. Further, for $f$ holomorphic in $|z|<R$ let

$$
M(f ; r):=\max _{|z|=r}|f(z)| \quad(0<r<R) .
$$

If $p$ belongs to $\mathscr{P}_{n}$ then so does $f(z):=z^{n} p(1 / z)$. As such, by the maximum modulus principle $M\left(f ; r^{-1}\right) \geq M(f ; 1)$ for $0<r<1$. Since $M\left(f ; r^{-1}\right)=$ $r^{-n} M(p ; r)$, we obtain

$$
M(p ; r) \geq r^{n} M(p ; 1) \quad \text { for } 0<r<1 .
$$

In (1) equality holds if and only if $p(z)$ is a constant multiple of $z^{n}$. It should, therefore, be possible to improve upon (1) if $p(z)$ does not vanish in $|z|<1$. Indeed, Rivlin [8] proved

Theorem A. Let $p \in \mathscr{P}_{n}$. If $p(z) \neq 0$ for $|z|<1$, then

$$
M(p ; r) \geq\left(\frac{1+r}{2}\right)^{n} M(p ; 1) \quad \text { for } 0<r<1 .
$$

The result is best possible with equality holding for polynomials of the form $(\lambda+\mu z)^{n},|\lambda|=|\mu|$.

Extensions of Theorem A were considered by Govil in [4]. After noting that (2) can be replaced by the somewhat more general inequality

$$
M(p ; r) \geq\left(\frac{1+r}{1+R}\right)^{n} M(p ; R) \quad \text { for } 0 \leq r<R \leq 1
$$

he proved.

Received by the editors July 12, 1990.

1991 Mathematics Subject Classification. Primary 30C10, 30C80, 30D15.

Key words and phrases. Polynomials, maximum modulus, entire functions of exponential type, Phragmén-Lindelöf indicator. 
Theorem B. Let $p \in \mathscr{P}_{n}$ and $p(z) \neq 0$ for $|z|<1$. If $p^{\prime}(0)=0$, then for $0 \leq r<R \leq 1$ we have

$$
M(p ; r) \geq\left(\frac{1+r}{1+R}\right)^{n}\left\{1 /\left(1-\frac{(1-R)(R-r) n}{4}\left(\frac{1+r}{1+R}\right)^{n-1}\right)\right\} M(p ; R) .
$$

Whereas (3) is sharp, inequality (4) leaves something to be desired. We are indeed able to replace it by

$$
M(p ; r) \geq\left(\frac{1+r^{2}}{1+R^{2}}\right)^{n / 2} M(p ; R) \quad \text { for } 0 \leq r<R \leq 1 .
$$

This inequality is sharp at least for even $n$ as is seen by considering polynomials of the form $\left(\lambda+\mu z^{2}\right)^{n / 2},|\lambda|=|\mu|$.

Our approach to the problem gives the two inequalities (3) and $\left(4^{\prime}\right)$ at the same time. The idea is of a wider scope, but in our presentation we shall prefer clarity and simplicity over generality. Nevertheless, we shall clearly indicate the extensions that can be obtained without much difficultly. We shall also present an application of Theorem $\mathrm{A}$.

A reader wondering about the value of a condition like " $p(0)=0$ " appearing in the statement of Theorem B might find some of the sections in $[9, \S 6$ in particular; 10; 7] persuasive. We wish to add that if $p(z):=\sum_{\nu=0}^{n} c_{\nu} z^{\nu}$ satisfies the conditions of Theorem A then $P(z):=p\left(z^{2}\right)$ belongs to $\mathscr{P}_{2 n}$ and satisfies the other two conditions of Theorem B. Hence according to $\left(4^{\prime}\right)$, if $0 \leq r<$ $R \leq 1$ then

$$
M(p ; r)=M(P ; \sqrt{r}) \geq\left(\frac{1+r}{1+R}\right)^{n} M(P ; \sqrt{R})=\left(\frac{1+r}{1+R}\right)^{n} M(p ; R),
$$

i.e., (3) can be seen as a corollary of $\left(4^{\prime}\right)$.

\section{A LEMMA}

We need the following auxiliary result.

Lemma 1. If $p(z):=c_{0}+\sum_{\nu=m}^{n} c_{\nu} z^{\nu}$ has all its zeros in $|z| \geq k>1$, then

$$
M\left(p^{\prime} ; 1\right) \leq \frac{n}{1+k^{m}} M(p ; 1)
$$

more precisely

$$
M\left(p^{\prime} ; 1\right) \leq n \frac{1+(m / n)\left|c_{m} / c_{0}\right| k^{m+1}}{1+k^{m+1}+(m / n)\left|c_{m} / c_{0}\right|\left(k^{m+1}+k^{2 m}\right)} M(p ; 1) .
$$

Remark 1. If $1+\gamma_{m} z^{m}+\cdots+\gamma_{n} z^{n} \neq 0$ for $|z|<1$, then [7, Theorem $2^{\prime}$ ] $\left|\gamma_{m}\right| \leq n / m$. Applying this result to the polynomial $p(k z) / c_{0}$ we conclude that

$$
\frac{m}{n}\left|\frac{c_{m}}{c_{0}}\right| k^{m} \leq 1
$$

Hence (5) is contained in (6).

Proof of Lemma 1. It follows from a well-known theorem of Laguerre (see, e.g.,

[6]) that if $p(z) \neq 0$ for $|z|<k$ then

$$
n p(z)-z p^{\prime}(z) \neq-\zeta p^{\prime}(z) \quad \text { for }|\zeta|<k,|z|<k .
$$


Hence

The function

$$
\left|\frac{p^{\prime}(z)}{n p(z)-z p^{\prime}(z)}\right| \leq \frac{1}{k} \quad \text { for }|z| \leq k
$$

$$
f(z):=\frac{k p^{\prime}(k z)}{n p(k z)-k z p^{\prime}(k z)}
$$

therefore, is, holomorphic in $|z| \leq 1$ where it satisfies $|f(z)| \leq 1$. Further, $f(0)=\cdots=f^{(m-1)}(0)=0$ and $f^{(m)}(0)=(m / n)\left(c_{m} / c_{0}\right) k^{m}$. Hence by a generalized form of Schwarz's lemma

$$
|f(z)| \leq|z|^{m-1} \frac{|z|+(m / n)\left|c_{m} / c_{0}\right| k^{m}}{(m / n)\left|c_{m} / c_{0}\right| k^{m}|z|+1}
$$

for $|z|<1$. In particular, this holds for $|z|=1 / k$ and so

$$
\left|p^{\prime}(z)\right| \leq \frac{1}{k^{m+1}} \frac{1+(m / n)\left|c_{m} / c_{0}\right| k^{m+1}}{(m / n)\left|c_{m} / c_{0}\right| k^{m-1}+1}\left|n p(z)-z p^{\prime}(z)\right| \quad \text { for }|z|=1 .
$$

But if $p \in \mathscr{P}_{n}$ then for $|z|=1$ the quantity $\left|n p(z)-z p^{\prime}(z)\right|$ does not exceed (see, e.g. [5, Lemma 1, p. 8]) $n M(p ; 1)-\left|p^{\prime}(z)\right|$ and therefore (6) holds.

The case $m=1$ of (6) was proved in [3]. Here we need a more general form of Schwarz's lemma; otherwise the proof is not very different.

\section{EXTENSIONS OF THEOREM A}

3.1. Our main result is

Theorem 1. If $p(z):=c_{0}+\sum_{\nu=m}^{n} c_{\nu} z^{\nu}$ has no zeros in $|z|<1$ then for $0 \leq r<$ $R \leq 1$

$$
M(p ; r) \geq\left(\frac{1+r^{m}}{1+R^{m}}\right)^{n / m} M(p ; R)
$$

more precisely

(8) $M(p ; r) \geq \exp \left(-n \int_{r}^{R} \frac{t^{m}+(m / n)\left|c_{m} / c_{0}\right| t^{m-1}}{t^{m+1}+(m / n)\left|c_{m} / c_{0}\right|\left(t^{m}+t\right)+1} d t\right) M(p ; R)$.

Proof. For $0 \leq r<R \leq 1$ and $\theta \in[0,2 \pi)$ we have

$$
\left|p\left(R e^{i \theta}\right)\right| \leq\left|p\left(r e^{i \theta}\right)\right|+\int_{r}^{R}\left|p^{\prime}\left(t e^{i \theta}\right)\right| d t,
$$

which readily implies

$$
M(p ; R) \leq M(p ; r)+\int_{r}^{R} M\left(p^{\prime} ; t\right) d t
$$

Since $p(z) \neq 0$ for $|z|<1, p(t z) \neq 0$ for $|z|<1 / t$ and so by (5)

$$
\max _{|z|=1} t\left|p^{\prime}(t z)\right| \leq \frac{n}{1+t^{-m}} \max _{|z|=1}|p(t z)|,
$$

which is equivalent to

$$
M\left(p^{\prime} ; t\right) \leq \frac{n t^{m-1}}{t^{m}+1} M(p ; t)
$$


Using this estimate in (9) we obtain

$$
M(p ; R) \leq M(p ; r)+\int_{r}^{R} \frac{n t^{m-1}}{t^{m}+1} M(p ; t) d t .
$$

Now let us denote the right-hand side of this inequality by $\Phi(R)$. Then $\Phi^{\prime}(R)=$ $\left(\left(n R^{m-1}\right) /\left(1+R^{m}\right)\right) M(p ; R)$ and (11) can be written in the form

$$
\Phi^{\prime}(R)-\frac{n R^{m-1}}{1+R^{m}} \Phi(R) \leq 0
$$

Multiplying through by $\left(1+R^{m}\right)^{-n / m}$ we obtain

$$
\left.\frac{d}{d R}\left(1+R^{m}\right)^{-n / m} \Phi(R)\right\} \leq 0,
$$

which implies that $\left(1+R^{m}\right)^{-n / m} \Phi(R)$ is a nonincreasing function of $R$ in $(0,1)$. Thus

$$
\Phi(r) \geq\left(\frac{1+r^{m}}{1+R^{m}}\right)^{n / m} \Phi(R) \quad \text { for } 0 \leq r<R \leq 1 .
$$

From this (7) follows since $\Phi(r)=M(p ; r)$ and $\Phi(R) \geq M(p ; R)$.

If we had used (6) instead of (5) we would have ended up with (8).

3.2.From (8) it follows, in particular, that if $p(z):=c_{0}+c_{1} z+\cdots+c_{n} z^{n} \neq 0$ for $|z|<1$, then for $0 \leq r<R \leq 1$

$$
\begin{aligned}
M(p ; r) & \geq \exp \left(-n \int_{r}^{R} \frac{t+(1 / n)\left|c_{1} / c_{0}\right|}{t^{2}+(2 / n)\left|c_{1} / c_{0}\right| t+1} d t\right) M(p ; R) \\
& =\left(\frac{1+(2 / n)\left|c_{1} / c_{0}\right| r+r^{2}}{1+(2 / n)\left|c_{1} / c_{0}\right| R+R^{2}}\right)^{n / 2} M(p ; R) .
\end{aligned}
$$

Thus we obtain the following extension of Theorem A, which also includes $\left(4^{\prime}\right)$.

Corollary 1. If $p(z):=\sum_{\nu=m}^{n} c_{\nu} z^{\nu}$ has no zeros in $|z|<1$ then for $0 \leq r<$ $R \leq 1$ we have

$$
M(p ; r) \geq\left(\frac{1+2|\lambda| r+r^{2}}{1+2|\lambda| R+R^{2}}\right)^{n / 2} M(p ; R),
$$

where $\lambda:=c_{1} / n c_{0}$.

3.3. Extension of Theorem $A$ to entire functions of exponential type. An entire function $f$ is said to be of exponential type $\tau$ if it is either of order less than 1 or it is of order 1 type at most $\tau$. The Phragmén-Lindelöf indicator of a function $f$ of exponential type is defined to be

$$
h_{f}(\theta):=\limsup _{r \rightarrow \infty} \frac{\log \left|f\left(r e^{i \theta}\right)\right|}{r} \quad(0 \leq \theta<2 \pi) .
$$

The boundedness of an entire function of exponential type on one line carries with it boundedness on every parallel line. Assuming that $f$ is bounded on the real axis we set

$$
\mu(f ; y):=\sup _{-\infty<x<\infty}|f(x+i y)|
$$


If $p \in \mathscr{P}_{n}$ then $f(z):=p\left(e^{i z}\right)$ is an entire function of exponential type $\tau=n$ bounded on the real axis. Besides, $h_{f^{\prime}}(\pi / 2) \leq-1, h_{g^{\prime}}(\pi / 2) \leq-1$ where $g(z):=e^{i \tau z} \overline{f(\bar{z})}$. If $p(z) \neq 0$ for $|z|<1$ then $f(z) \neq 0$ for $\operatorname{Im} z>0$ and $h_{f}(\pi / 2)=0$. Our extension of Theorem A to entire functions of exponential type reads as follows.

Theorem 2. Let $f$ be an entire function of exponential type $\tau$ bounded on the real axis. Further, let $h_{f}(\pi / 2)=0, h_{f^{\prime}}(\pi / 2) \leq-\lambda<0$, and $h_{g^{\prime}}(\pi / 2) \leq-\lambda$ where $g(z):=e^{i \tau z} \overline{f(\bar{z})}$. If $f(z) \neq 0$ for $\operatorname{Im} z>0$, then

$$
\mu(f ; y) \geq\left(\frac{1+e^{-\lambda y}}{1+e^{-\lambda \eta}}\right)^{\tau / \lambda} \mu(f ; \eta) \quad \text { for } 0 \leq \eta<y<\infty .
$$

Remark 2. Theorem 2 applies to functions of the form

$$
f(z):=c_{0}+\int_{\lambda}^{\tau-\lambda} e^{i z t} \varphi(t) d t+c e^{i \tau z} \quad(0<\lambda \leq \tau / 2, \varphi \in L(\lambda, \tau-\lambda))
$$

not vanishing in the upper half plane.

Proof of Theorem 2. For $t>0$ the function $f_{t}(z):=f(z+i t)$ satisfies all the conditions of Theorem 2 and is even $\neq 0$ in the larger half plane $\operatorname{Im} z>-t$. According to a known result [2, Theorem 3]

$$
\mu\left(f^{\prime} ; t\right)=\mu\left(f_{t}^{\prime} ; 0\right) \leq \frac{\tau}{1+e^{\lambda t}} \mu\left(f_{t} ; 0\right)=\frac{\tau}{1+e^{\lambda t}} \mu(f ; t),
$$

and so for $0 \leq \eta<y$ we have

$$
\mu(f ; \eta) \leq \mu(f ; y)+\int_{\eta}^{y} \frac{\tau}{1+e^{\lambda t}} \mu(f ; t) d t .
$$

Denoting the right-hand side of this inequality by $\psi(\eta)$ we obtain

$$
\psi^{\prime}(\eta)+\frac{\tau}{1+e^{\lambda \eta}} \psi(\eta) \geq 0
$$

which implies that $\left(1+e^{-\lambda \eta}\right)^{-\tau / \lambda} \psi(\eta)$ is a nondecreasing function of $\eta$ for $\eta \geq 0$. Hence (16) holds.

\section{An APPLICATION}

For $R>1$ let $\mathscr{E}_{R}$ denote the ellipse whose foci are $-1,+1$ and the sum of whose semiaxes is $R$. If $p \in \mathscr{P}_{n}$ and $p(x)$ is real for real $x$, then [1]

$$
\max _{z \in \mathscr{E}_{R}}|p(z)| \leq\left(R^{n}+R^{-n}\right) / 2 \max _{-1 \leq x \leq 1}|p(x)| .
$$

In (17) equality holds for constant multiples of $T_{n}$ (the $n$th Chebyshev polynomial of the first kind), which, as we know, has all its zeros on the interval $(-1,1)$. We prove

Theorem 3. If $p(z):=\sum_{\nu=m}^{n} c_{\nu} z^{\nu}$ has all its zeros on the interval $[-1,1]$, then

$$
\max _{z \in \mathscr{E}_{R}}|p(z)| \geq\left(\frac{R^{2}+2|\Lambda| R+1}{2(|\Lambda|+1) R}\right)^{n} \max _{-1 \leq x \leq 1}|p(x)|
$$

where $\Lambda:=c_{n-1} / n c_{n}$. 
Proof. Since $T_{n}(x)=2^{n-1} x^{n}+t_{n-2}(x)$ where $t_{n-2} \in \mathscr{P}_{n-2}$, we have

$$
p(x)=\frac{1}{2^{n-1}} c_{n} T_{n}(x)+\frac{1}{2^{n-2}} c_{n-1} T_{n-1}(x)+\sum_{k=2}^{n} d_{k} T_{n-k}(x)
$$

and so

$$
\begin{aligned}
p\left(\frac{z+z^{-1}}{2}\right)= & \frac{1}{2^{n-1}} c_{n} \frac{z^{n}+z^{-n}}{2}+\frac{1}{2^{n-2}} c_{n-1} \frac{z^{n-1}+z^{-n+1}}{2} \\
& +\frac{1}{2} \sum_{k=2}^{n} d_{k}\left(z^{n-k}+z^{-n+k}\right) .
\end{aligned}
$$

Thus

$$
f(z):=z^{n} p\left(\frac{z+z^{-1}}{2}\right)=\frac{1}{2^{n}} c_{n}+\frac{1}{2^{n-1}} c_{n-1} z+\cdots+\frac{1}{2^{n}} c_{n} z^{2 n}
$$

is a polynomial of degree $2 n$ having all its zeros on $|z|=1$. So $|\Lambda| \leq 1$ and by Theorem 2

$$
M\left(f ; R^{-1}\right) \geq\left(\frac{1+2|\Lambda| R^{-1}+R^{-2}}{2(|\Lambda|+1)}\right)^{n} M(f ; 1) .
$$

But $M\left(f ; R^{-1}\right)=R^{-n} \max _{z \in \mathscr{C}_{R}}|p(z)|, M(f ; 1)=\max _{-1 \leq x \leq 1}|p(x)|$ and hence (18) holds.

Since $|\Lambda| \leq 1$, we obtain, in particular, that if a polynomial $p$ belonging to $\mathscr{P}_{n}$ has all its zeros on $[-1,1]$, then for all $R>1$

$$
\max _{z \in \mathscr{E}_{R}}|p(z)| \geq\left(\frac{R^{1 / 2}+R^{-1 / 2}}{2}\right)^{2 n} \max _{-1 \leq x \leq 1}|p(x)| .
$$

It is easily seen that in (20) equality holds for constant multiples of

$$
p^{*}(z):=\left(\begin{array}{c}
2 n \\
n
\end{array}\right)+2 \sum_{k=1}^{n}\left(\begin{array}{c}
2 n \\
n-k
\end{array}\right) z^{k}
$$

and, of course, also for those of the polynomial $z \mapsto p^{*}(-z)$.

\section{REFERENCES}

1. R. J. Duffin and A. C. Schaeffer, Some properties of functions of exponential type, Bull. Amer. Math. Soc. 44 (1938), 236-240.

2. N. K Govil and Q. I. Rahman, Functions of exponential type not vanishing in a half plane and related polynomials, Trans. Amer. Math. Soc. 137 (1969), 501-517.

3. N. K. Govil, Q. I. Rahman, and G. Schmeisser, On the derivative of a polynomial, Illinois J. Math. 23 (1979), 319-329.

4. N. K. Govil, On the maximum modulus of polynomials, J. Math. Anal. Appl. 112 (1985), 253-258.

5. Q. I. Rahman, Applications of functional analysis to extremal problems for polynomials, Sém. Math. Sup. été 1967, Les Presses de l'Université de Montréal, 291968.

6. Q. I. Rahman and G. Schmeisser, Extension of a theorem of Laguerre to entire functions of exponential type, J. Math. Anal. Appl. 122 (1987), 463-468.

7. Q. I. Rahman and J. Stankiewicz, Differential inequalities and local valency, Pacific J. Math. 54 (1974), 165-181. 
8. T. J. Rivlin, On the maximum modulus of polynomials, Amer. Math. Monthly 67 (1960), 251-253.

9. J. L. Walsh, The location of the zeros of the derivative of a rational function, revisited, J. Math. Pures Appl. 43 (1964), 353-370.

10. _ A theorem of Grace on the zeros of polynomials, revisited, Proc. Amer. Math. Soc. 15, (1964), 354-360.

736 Cote-STe-Catherine, Montréal, Québec, H3T 1A3 Canada 